Article

\title{
Coordinated Localization and Antagonistic Function of NtPLC3 and PI4P 5-Kinases in the Subapical Plasma Membrane of Tobacco Pollen Tubes
}

\author{
Irene Stenzel ${ }^{1}$, Till Ischebeck ${ }^{2} \mathbb{1}$, Linh Hai Vu-Becker ${ }^{1}$, Mara Riechmann ${ }^{1}$, \\ Praveen Krishnamoorthy ${ }^{1}\left(\mathbb{D}\right.$, Marta Fratini ${ }^{1}$ (D) and Ingo Heilmann ${ }^{1, *}$ \\ 1 Department of Cellular Biochemistry, Institute for Biochemistry and Biotechnology, Martin Luther \\ University Halle-Wittenberg, 06120 Halle (Saale), Germany; irene.stenzel@biochemtech.uni-halle.de (I.S.); \\ linhvu@gmx.de (L.H.V.-B.); mriechmann@biochem.uni-kiel.de (M.R.); \\ praveen.krishnamoorthy@wustl.edu (P.K.); marta.fratini@bct.uni-halle.de (M.F.) \\ 2 Department of Plant Biochemistry, Albrecht-von-Haller-Institute of Plant Sciences and Göttingen Center for \\ Molecular Biosciences (GZMB), Georg August University Göttingen, 37077 Göttingen, Germany; \\ tischeb@gwdg.de \\ * Correspondence: ingo.heilmann@biochemtech.uni-halle.de; Tel.: +49-345-55-24840
}

Received: 12 March 2020; Accepted: 1 April 2020; Published: 3 April 2020

\begin{abstract}
Polar tip growth of pollen tubes is regulated by the membrane phospholipid phosphatidylinositol 4,5-bisphosphate $\left(\operatorname{Ptd} \operatorname{Ins}(4,5) \mathrm{P}_{2}\right)$, which localizes in a well-defined region of the subapical plasma membrane. How the PtdIns $(4,5) \mathrm{P}_{2}$ region is maintained is currently unclear. In principle, the formation of PtdIns $(4,5) \mathrm{P}_{2}$ by PI4P 5-kinases can be counteracted by phospholipase $\mathrm{C}$ (PLC), which hydrolyzes PtdIns $(4,5) \mathrm{P}_{2}$. Here, we show that fluorescence-tagged tobacco NtPLC3 displays a subapical plasma membrane distribution which frames that of fluorescence-tagged PI4P 5-kinases, suggesting that NtPLC3 may modulate PtdIns $(4,5) \mathrm{P}_{2}$-mediated processes in pollen tubes. The expression of a dominant negative NtPLC3 variant resulted in pollen tube tip swelling, consistent with a delimiting effect on $\operatorname{Ptd} \operatorname{Ins}(4,5) \mathrm{P}_{2}$ production. When pollen tube morphologies were assessed as a quantitative read-out for PtdIns $(4,5) \mathrm{P}_{2}$ function, NtPLC3 reverted the effects of a coexpressed PI4P 5-kinase, demonstrating that NtPLC3-mediated breakdown of PtdIns $(4,5) \mathrm{P}_{2}$ antagonizes the effects of PtdIns $(4,5) \mathrm{P}_{2}$ overproduction in vivo. When analyzed by spinning disc microscopy, fluorescence-tagged NtPLC3 displayed discontinuous membrane distribution omitting punctate areas of the membrane, suggesting that NtPLC3 is involved in the spatial restriction of plasma membrane domains also at the nanodomain scale. Together, the data indicate that NtPLC3 may contribute to the spatial restriction of $\operatorname{PtdIns}(4,5) \mathrm{P}_{2}$ in the subapical plasma membrane of pollen tubes.
\end{abstract}

Keywords: Phosphatidylinositol 4,5-bisphosphate; PtdIns(4,5) $\mathrm{P}_{2}$; phospholipase C; PI4P 5-kinase; pollen tube tip swelling

\section{Introduction}

Pollen tubes are tip-growing cells with a key function in plant sexual reproduction [1]. The elongation of pollen tubes is strictly limited to the cells' apex, from where cell wall material is secreted [2-5]. In the tip of the growing pollen tube, secretion, membrane trafficking and cytoskeletal elements are controlled in part by the membrane phospholipid phosphatidylinositol 4,5-bisphosphate (PtdIns $\left.(4,5) \mathrm{P}_{2}\right)[3,6]$. To exert its regulatory functions, PtdIns $(4,5) \mathrm{P}_{2}$ binds to cytoplasmic proteins possessing specific lipid binding domains, which are recruited to the plasma membrane by their lipid ligand $[7,8]$. The recruitment of $\operatorname{PtdIns}(4,5) \mathrm{P}_{2}$-binding proteins is thought to underlie the dynamic plasma membrane association of the protein complexes required for cell expansion $[3,9,10]$. 
PtdIns $(4,5) \mathrm{P}_{2}$ is formed by PI4P 5-kinases, which are encoded in the Arabidopsis genome by a family of eleven genes [11]. Based on their domain structure, PI4P 5-kinases can be categorized in subfamilies A (isoforms PIP5K10 and PIP5K11) and B (isoforms PIP5K1-PIP5K9), and the isoforms PIP5K2, PIP4K4, PIP5K5, PIP5K6, PIP5K10 and PIP5K11 are expressed in pollen and pollen tubes [7,8]. The importance of PtdIns $(4,5) \mathrm{P}_{2}$ formation for pollen tube growth is underlined by the observation that Arabidopsis mutants with defects in genes encoding PI4P 5-kinases display reduced pollen germination, and diminished pollen tube growth for cells that succeed in emerging, including pip $5 k 4$ pip5k5 double mutants and pip5k10 pip5k11 double mutants [12-15].

In line with its important function in controlling polar tip-growth, fluorescent probes for $\operatorname{PtdIns}(4,5) \mathrm{P}_{2}$ indicate an enrichment of this lipid in a subapical plasma membrane region of pollen tubes which extends from 2-3 $\mu \mathrm{m}$ to approximately $25 \mu \mathrm{m}$ below the pollen tube apex [12-17]. The distribution pattern of the PtdIns $(4,5) \mathrm{P}_{2}$ biosensors is largely mirrored by the distribution of fluorescence-tagged PI4P 5-kinases in these cells, and it has been proposed that the spatial positioning of PI4P 5-kinases is a factor controlling the extent of PtdIns $(4,5) \mathrm{P}_{2}$ in the plasma membrane of pollen tubes $[12,13,18]$. The spatial dimensions of the PtdIns $(4,5) \mathrm{P}_{2}$ domain in the subapical plasma membrane are critical for polar tip-growth, as evidenced by the observation that the overproduction of $\operatorname{Ptd} \operatorname{Ins}(4,5) \mathrm{P}_{2}$, upon overexpression of PI4P 5-kinases, and the ensuing enlargement of the plasma membrane domain occupied by this lipid results in altered patterns of pollen tube expansion [12,13]. A loss of apical cell polarity upon overproduction of $\operatorname{Ptd} \operatorname{Ins}(4,5) \mathrm{P}_{2}$ gives rise to substantial changes in pollen tube cell shape, including apical tip swelling $[13,19]$ or in more complex cases, branched cell morphologies $[12,14,16,19]$. From these observations, it is clear that the extent of the subapical $\operatorname{PtdIns}(4,5) \mathrm{P}_{2}$ domain must be tightly controlled to enable "normal" pollen tube growth.

Besides the biosynthesis of $\operatorname{Ptd} \operatorname{Ins}(4,5) \mathrm{P}_{2}$, its degradation can also contribute to defining its spatial distribution in the apical plasma membrane of pollen tubes. PtdIns $(4,5) \mathrm{P}_{2}$ can be hydrolyzed by phospholipase C (PLC) to form diacylglycerol (DAG) and inositol 1,4,5-trisphosphate ( $\mathrm{InsP}_{3}$ ), thus removing $\operatorname{Ptd} \operatorname{Ins}(4,5) \mathrm{P}_{2}$ as a polarized recruitment signal from the plasma membrane $[7,8]$. The Arabidopsis genome encodes nine isoforms of PLC [8,11], four of which are expressed in pollen. So far, the role for PLCs in Arabidopsis pollen tube growth has not been analyzed in detail. In previous experiments, it was shown that a fluorescence-tagged variant of the tobacco (Nicotiana tabacum) PLC, NtPLC3, displays a subapical plasma membrane distribution in tobacco pollen tubes [20], which at first approximation appears similar to that observed for PI4P 5-kinases. A fluorescence-tagged variant of PLC1 from petunia (Petunia inflata) displayed an equivalent localization pattern when expressed in petunia pollen tubes [21]. Both NtPLC3 and PLC1 from petunia are active phosphoinositide-specific PLCs and most similar to the PLC $\zeta$ family [22]. Interestingly, the expression of a dominant negative variant of petunia PLC1 resulted in substantial pollen tube tip swelling [21], consistent with delimited $\operatorname{PtdIns}(4,5) \mathrm{P}_{2}$ formation upon reduced hydrolysis by the catalytically inactive PLC1 from petunia.

While the localization and cell morphology data suggest that PLCs may serve as $\operatorname{PtdIns}(4,5) \mathrm{P}_{2}$-degrading enzymes in pollen tubes and functionally antagonize $\mathrm{Ptd} \operatorname{Ins}(4,5) \mathrm{P}_{2}$ formation by PI4P 5-kinases, there has been no dedicated analysis of the functional interplay of PLCs and PI4P 5-kinases to date. The pollen tube system is an ideal model to study the functional interrelations of PLC with PI4P 5-kinase because of the well-defined subapical plasma membrane domain, which is easy to monitor by confocal microscopy, and because of the well-characterized cell morphologies ensuing from the overproduction of $\operatorname{Ptd} \operatorname{Ins}(4,5) \mathrm{P}_{2}$.

Here, we demonstrate that fluorescence-tagged NtPLC3 displays a subapical plasma membrane distribution which encompasses a slightly narrower plasma membrane region decorated by the PI4P 5-kinases PIP5K2 or PIP5K11. A catalytically inactive variant of NtPLC3 exerts a substantial dominant negative effect when expressed in pollen tubes, which closely reflects the effect of overexpressed PIP5K2. In coexpression experiments, NtPLC3 functionally antagonizes the effects of overexpressed PIP5K2, resulting in a large proportion of normal growing pollen tubes upon coexpression of both enzymes. Together with a plasma membrane distribution in a discontinuous pattern omitting punctate 
areas determined by spinning disc (SD) analysis, our data suggest that NtPLC3 may contribute to the spatial restriction of $\operatorname{PtdIns}(4,5) \mathrm{P}_{2}$ in the plasma membrane of pollen tubes.

\section{Results}

PtdIns $(4,5) \mathrm{P}_{2}$ in the subapical plasma membrane domain of pollen tubes is formed by PI4P 5-kinases and can be degraded by PLC. Here, we address the interplay of these enzymes to balance $\operatorname{Ptd} \operatorname{Ins}(4,5) \mathrm{P}_{2}$ biosynthesis and degradation

\subsection{NtPLC3 Localizes to A Subapical Membrane Domain Encompassing Domains Occupied by PIP5K2 or PIP5K11.}

It has previously been reported that the distribution of $\operatorname{PtdIns}(4,5) \mathrm{P}_{2}$ in pollen tubes, as indicated by fluorescent biosensors such as RedStar-PLC81-PH, closely matches the distribution of PI4P 5-kinases [12, 13]; a similar pattern was also reported for fungal hyphae [23]. The relative extent of the subapical plasma membrane regions decorated by RedStar-PLC $81-\mathrm{PH}$ or by PIP5K11 was verified for tobacco pollen tubes in coexpression experiments (Figure 1A). To prevent morphological changes which PIP5K11 overexpression might cause, expression levels were kept low. The observation that the plasma membrane region marked by the $\operatorname{Ptd} \operatorname{Ins}(4,5) \mathrm{P}_{2}$ biosensor does not extend beyond its site of biosynthesis suggested that the lateral diffusion of $\operatorname{PtdIns}(4,5) \mathrm{P}_{2}$ might be limited, possibly by a PtdIns $(4,5) \mathrm{P}_{2}$-degrading enzyme such as PLC. Therefore, the relative spatial distributions of PI4P 5-kinases and PLC in the subapical plasma membrane region of tobacco pollen tubes was analyzed in coexpression experiments (Figure 1B-E). The previously reported NtPLC3 was selected for this analysis because it is well characterized and has been shown to occupy a subapical plasma membrane domain in tobacco pollen tubes [20]. This pattern was compared to the distribution of the respectively coexpressed PI4P 5-kinases PIP5K2 [19,24-26] and PIP5K11 [13], which represent the two subfamilies, i.e., A and B, of Arabidopsis PI4P 5-kinases [11]. When a PIP5K2-mCherry fusion was coexpressed at low levels with EYFP-NtPLC3, both enzymes decorated subapical plasma membrane domains (Figure 1B), with the noted distinction that the membrane domain occupied by EYFP-NtPLC3 was elongated over the subapical membrane region and spanned the entire region occupied by AtPIP5K2-mCherry. PIP5K2-mCherry localized more to the pollen tube tip with some signal in the subapical region, whereas EYFP-NtPLC3 localized mainly to the pollen tube shank. When EYFP-PIP5K11 was coexpressed with NtPLC3-RFP, the NtPLC-RFP marker spanned a larger plasma membrane domain encompassing the region decorated by EYFP-PIP5K11 (Figure 1C) in a pattern resembling that observed for PIP5K2-mCherry and EYFP-NtPLC3 (Figure 1A). The dimensions of the subapical plasma membrane regions occupied by NtPLC3-RFP or EYFP-PIP5K11 were monitored over time, as indicated by the durations in the individual panels of selected frames (Figure 1C). A two-channel kymograph analysis over a period of $10 \mathrm{~min}$ at a frame rate of $\sim 2$ frames per minute indicated that the distance of the occupied areas from the growing pollen tube tip remained roughly constant (Figure 1D). The fast growth rate and nonlinear growth of the pollen tubes required manual adjustment to obtain the kymographs. To provide an independent analysis of the relative distribution patterns of NtPLC3 and PIP5K11, the intensity and distribution of the PIP5K11 and PLC signals were additionally analyzed using the machine learning program Ilastik [27], minimizing bias (Figure 1E). The resulting very similar pattern suggests that NtPLC3 continuously accompanies PI4P 5-kinases in the subapical plasma membrane and towards the shank of the pollen tube, thereby possibly preventing the uncontrolled diffusion of $\operatorname{PtdIns}(4,5) \mathrm{P}_{2}$ far from its sites of biosynthesis [28]. 

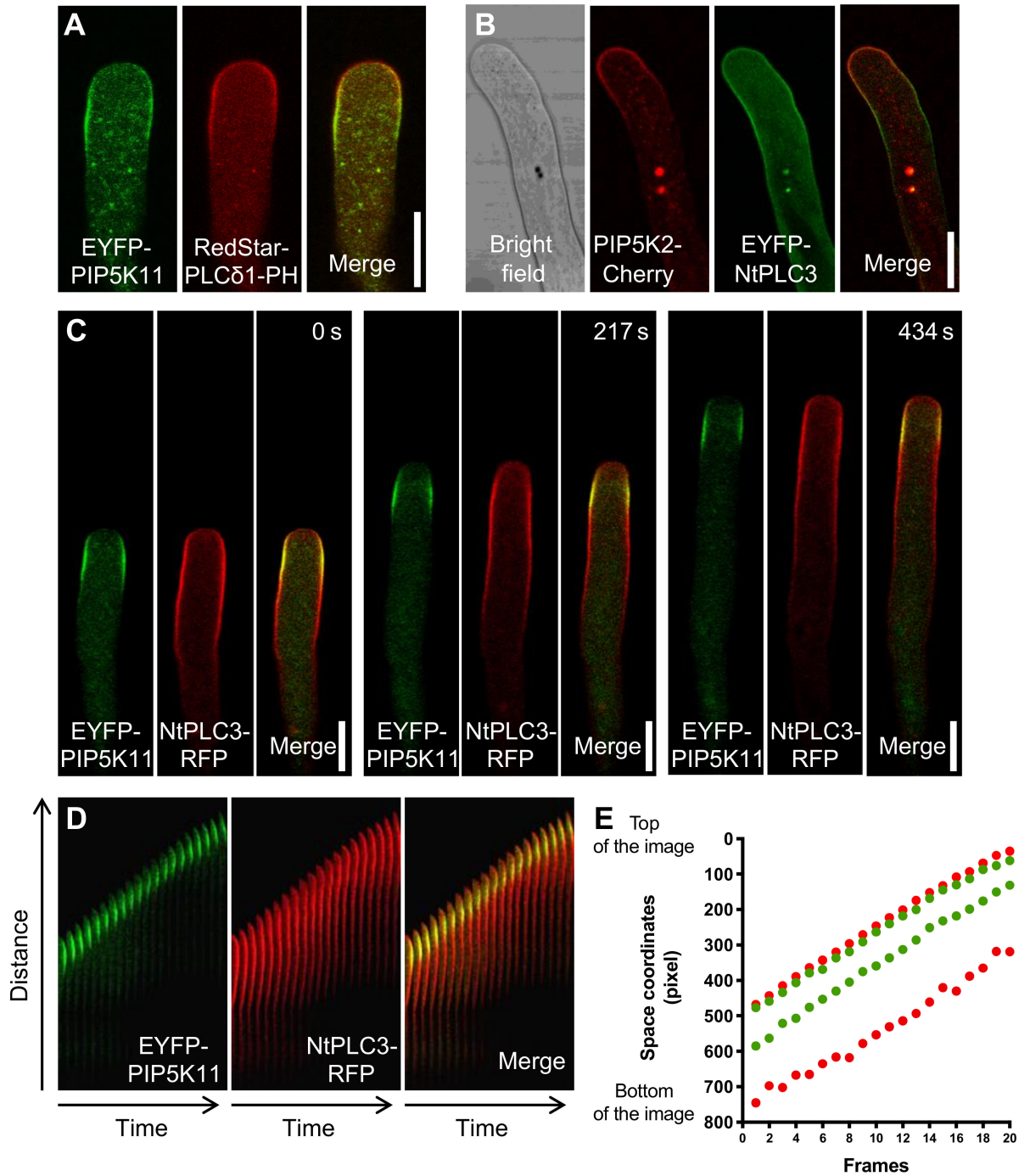

Figure 1. NtPLC3 localizes to a subapical plasma membrane domain encompassing domains occupied by PIP5K2 or PIP5K11. The localization of NtPLC3 was monitored by confocal imaging upon transient expression in tobacco pollen tubes. For these localization studies, expression levels were kept low to minimize morphological changes of the pollen tube cells. (A) Coexpression of EYFP-PIP5K11 with RedStar-PLC $81-\mathrm{PH}$, a fluorescent biosensor for PtdIns $(4,5) \mathrm{P}_{2}$, as indicated. B-E, Association of fluorescence-tagged NtPLC3 with an extended subapical plasma membrane domain, which spans the region occupied by coexpressed PIP5K2-mCherry or EYFP-PIP5K11. (B) Coexpression of EYFP-NtPLC3 with PIP5K2-mCherry. (C) Coexpression of NtPLC3-RFP with EYFP-PIP5K11, monitored in a time lapse experiment. The time series shown was recorded for $600 \mathrm{~s}$ at a frame rate of 2.14 frames $\mathrm{min}^{-1}$. Three selected frames are shown, as indicated. (D) Kymograph analysis of median confocal LSM sections of a growing pollen tube from (C), indicating the dynamic relative movements of PIP5K2-EYFP (green) and NtPLC3-RFP (red). Right panel: merged images (overlap indicated by yellow). (E) Intensity and distribution of the EYFP-PIP5K11 (green) and NtPLC3-RFP (red) signals were further analyzed by the machine learning program Ilastik to provide nonbiased results. Note that the relative distances from the pollen tube tip are roughly constant, and that the region occupied by NtPLC3-RFP continuously spans the region occupied by PIP5K2-EYFP. Data are representative for five independent experiments. Scale bars $=10 \mu \mathrm{m}$.

\subsection{Expression of Dominant Negative NtPLC3-RFP H124A Results in Pollen Tube Tip Swelling.}

To test the functional effects of NtPLC3 on pollen tube growth, we created a dominant negative variant, NtPLC3 H124A, in analogy to the previous report by Dowd and co-workers [21]. Both NtPLC3 
and NtPLC3 H124A were recombinantly expressed in Escherichia coli as fusions to an N-terminal maltose binding protein (MBP) tag for enhanced solubility and tested for activity (Figure 2). PLC activity was determined in vitro by monitoring the release of radiolabel from a hydrophobic $2\left[{ }^{3} \mathrm{H}\right] \mathrm{Ptd} \operatorname{Ins}(4,5) \mathrm{P}_{2}$ substrate from an organic into an aqueous solvent phase, as previously shown $[29,30]$. When the recombinant proteins (Figure 2A) were tested in vitro, the catalytic activity of MBP-NtPLC3 H124A protein was reduced by $99 \%$ compared to wild type MBP-NtPLC3 protein, and did not differ from that observed with the MBP negative control (Figure 2B). The biochemical tests enabled the use of the cataly tically inactive NtPLC3 H124A as a dominant negative variant of NtPLC3.

A

A
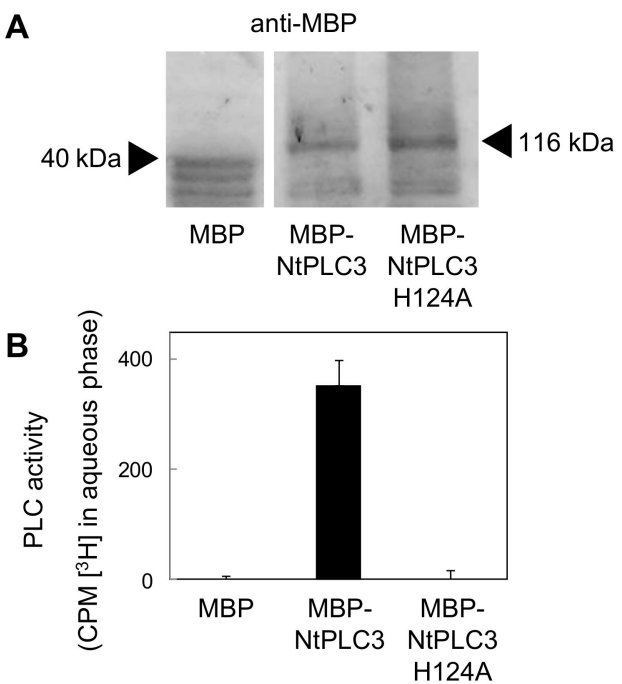

Figure 2. A catalytically inactive NtPLC3 variant, NtPLC3 H124A. A catalytically inactive variant of NtPLC3 to study dominant negative effects in vivo was generated by substituting histidine 124 for alanine. The effect of this substitution on enzyme function was tested using protein recombinantly produced in E. coli as a fusion to an N-terminal maltose-binding protein (MBP)-tag. (A) Immuno detection of recombinant MBP control, MBP-NtPLC3 and the dominant negative MBP-NtPLC3 H124A using an anti-MBP antibody, as indicated. (B) Catalytic activity of recombinant MBP, MBP-NtPLC3 and MBP-Nt-PLC3 H124A against a $2\left[{ }^{3} \mathrm{H}\right] \mathrm{PtdIns}(4,5) \mathrm{P}_{2}$ substrate in vitro. Data are the mean \pm SD from three experiments.

While experimental conditions for the localization studies shown in Figure 1 were chosen to ensure weak expression and normal cell growth, constructs were now intentionally overexpressed to test their effects on cell morphology. Overexpression of the dominant negative NtPLC3-RFP H124A in tobacco pollen tubes resulted in pollen tube tip swelling (Figure 3A), similar to the pattern previously reported for the expression of dominant negative Petunia PLC1 [21]. We assume that the overexpression of NtPLC-RFP H124A from the Lat52 promoter was strong enough to exert the dominant negative effect by displacing a substantial proportion of the intrinsic PLC3 population present in the pollen tube cells. The expression of EYFP or active NtPLC3 served as negative controls and resulted in pollen tube morphologies similar to those of untransformed pollen tubes (Figure 3A). The overexpression of NtPLC3-RFP H124A resulted in pollen tubes with tip diameters of up to $27 \mu \mathrm{m}$ (Figure 3B). This phenotypic effect was similar, although to a slightly lesser extent, to cell morphologies observed upon overexpression of PI4P 5-kinases, such as PIP5K2 or PIP5K11 (Figure 3B). No pollen tube tip swelling was observed upon overexpression of PIP5K5 (Figure 3A and B), which is consistent with previous reports stating that the expression of this enzyme results in overactive apical secretion and a tip branching phenotype [12,14]. To further characterize the effect of NtPLC3, the incidence of pollen tube morphologies previously observed upon overproduction of $\operatorname{Ptd} \operatorname{Ins}(4,5) \mathrm{P}_{2}$ was scored, including the analysis of tip branching and tip swelling (Figure 3C). 
A

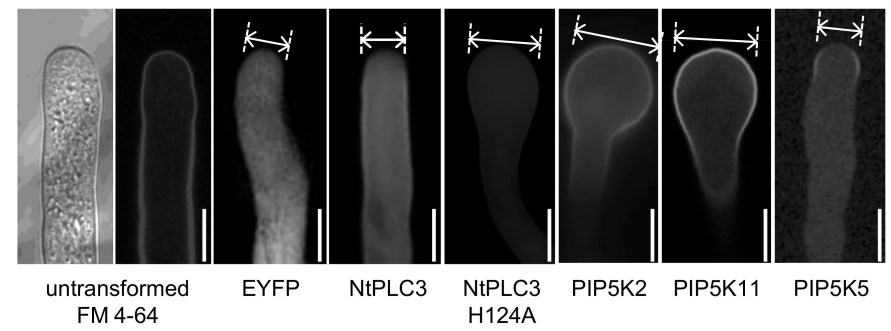

B

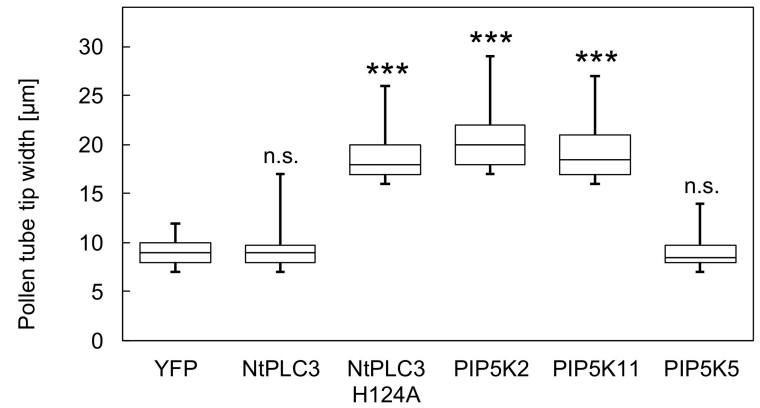

C

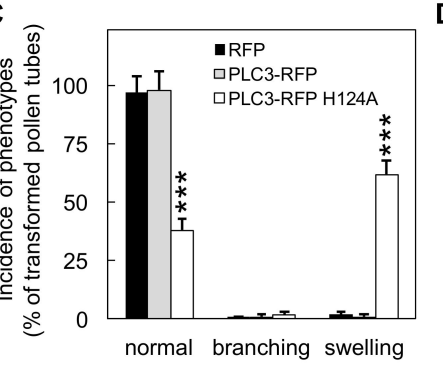

D

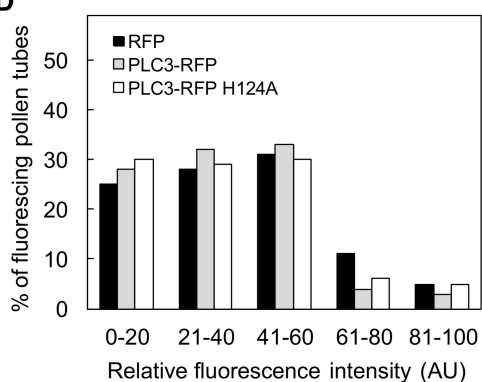

Figure 3. Overexpression of dominant negative RFP-NtPLC3 H124A induces pollen tube tip swelling, similar to expressed PI4P 5-kinases. The effects of NtPLC3 or NtPLC3 H124A on cell morphologies were tested upon transient overexpression in tobacco pollen tubes. The expression of all constructs was driven by the strong pollen-specific Lat52 promoter. (A) The extent of pollen tube tip swelling was assessed upon overexpression of NtPLC3, NtPLC3 H124A, PIP5K2, PIP5K11, or PIP5K5. Negative controls included untransformed pollen tubes, stained with the membrane dye, FM 4-64, or pollen tubes expressing EYFP, as indicated. (B) Pollen tube tip widths as determined for the respective expressed constructs. Asterisks indicate a significant difference to the YFP control according to a Student's t-test $\left.{ }^{* * *}, p \leq 0.01\right)$; n.s., not significant. Data are from at least five indepen dent experiments and $>50$ cells were analyzed for each transformation. (C) Based on the same data set, the incidence of normal, branched or swollen cell morphologies was determined upon transient expression of RFP, NtPLC3-RFP or NtPLC3-RFP H124A in tobacco pollen tubes. Note that overex pression of the dominant negative NtPLC3-RFP H124A mediated pollen tube tip swelling, not tip branching. Asterisks indicate a significant difference to the RFP control according to a Student's t-test $\left.{ }^{* * *}, p \leq 0.01\right)$. Data are from at least five independent experiments, and $>50$ cells were analyzed for each transformation. (D) Fluorescence intensities recorded during the expression of RFP, NtPLC3-RFP or NtPLC3-RFP H124A. Scale bars, $10 \mu \mathrm{m}$.

Interestingly, while pollen tube tip swelling was observed in $\sim 60 \%$ of cells expressing the dominant negative NtPLC-RFP H124A (Figure 3C), in a pattern similar to previous observations on petunia pollen tubes [21], no tip branching was observed. Tip branching was positively observed in our experiments upon overexpression of PIP5K5, indicating that branching could occur under the experimental conditions used. Differences in pollen tube morphologies between samples were not the result of different expression levels of the expressed proteins, as fluorescence intensities were similar (Figure 3D). While the intensity measurements indicate mean values for the pollen tube tip region, we observed enhanced cytoplasmic fluorescence in pollen tubes displaying tip swelling, as, for instance, 
upon overexpression of NtPLC3-RFP H124 A (Figure 3A). Considering further previous observations on tip swelling upon PtdIns $(4,5) \mathrm{P}_{2}$ overproduction $[19,31]$, the effects of the expression of dominant negative NtPLC3-RFP H124A are consistent with increased formation of PtdIns $(4,5) \mathrm{P}_{2}$, and suggest that NtPLC3 might antagonize PtdIns $(4,5) \mathrm{P}_{2}$ production mediated by PI4P 5-kinases.

\subsection{NtPLC3-RFP Antagonizes the Effects of Coexpressed PIP5K2-EYFP on Pollen Tube Tip Swelling.}

To elucidate the functional interplay of NtPLC3 with PIP5K2, we coexpressed AtPIP5K2-EYFP with either NtPLC3-RFP or NtPLC3-RFP H124A and quantified the ensuing effects on pollen tube morphologies (Figure 4). In these experiments, the overexpression of AtPIP5K2-EYFP resulted in $\sim 75 \%$ of cells displaying tip swelling (Figure $4 \mathrm{~A}$ ). This effect was abated upon coexpression of AtPIP5K2-EYFP with NtPLC3-RFP, but not upon coexpression with NtPLC3-RFP H124A (Figure 4A), indicating that NtPLC3-RFP counteracted the effects of overexpressed AtPIP5K2-EYFP. Coexpression of AtPIP5K2-EYFP with NtPLC3-RFP H124A resulted in a slight increase in tip swelling (Figure 4A), but no fully additive effect, suggesting that AtPIP5K2-EYFP and NtPLC3-RFP H124A may modulate the same pool of PtdIns $(4,5) \mathrm{P}_{2}$. No combination of the expressed proteins resulted in detectable pollen tube tip branching, and no substantial differences in the expression levels of the different proteins were observed in the transformed pollen tubes, based on the detected fluorescence intensities (Figure 4B). Together, the results support the hypothesis that NtPLC3 expression has an antagonistic effect on the pollen tube tip-swelling phenotype arising from AtPIP5K2 activity.
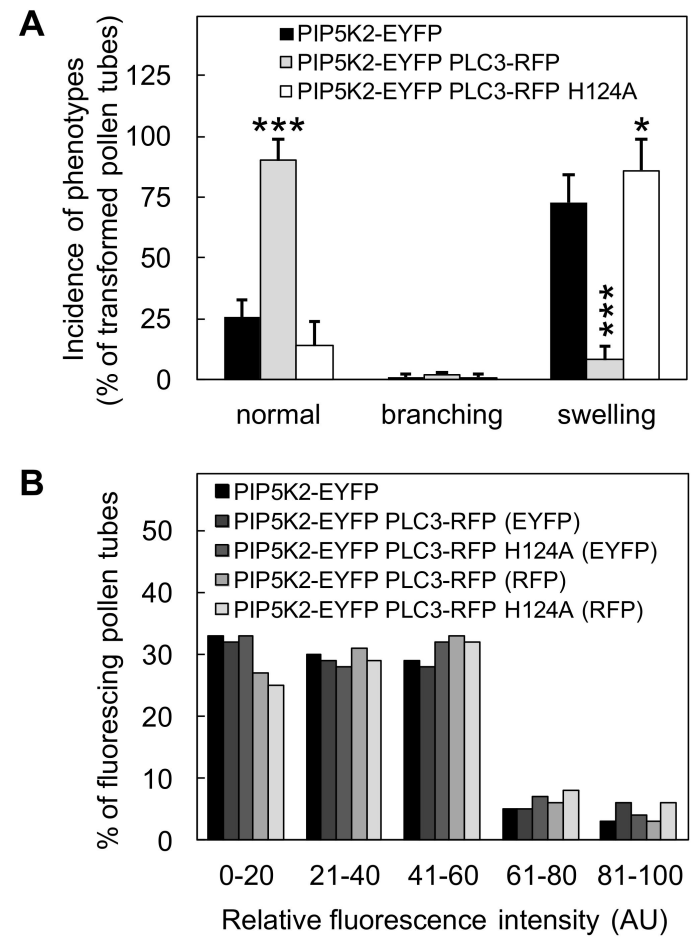

Figure 4. NtPLC3-RFP counteracts PIP5K2-EYFP-induced PtdIns(4,5) $\mathbf{P}_{2}$-dependent tip swelling. The effects of NtPLC3 on cell morphologies mediated by the overexpression of PIP5K2 were tested upon transient co-overexpression in tobacco pollen tubes. The expression of all constructs was driven by the strong pollen-specific Lat52 promoter. (A) Incidence of normal, branched or swollen cell morphologies upon transient overexpression of AtPIP5K2-EYFP alone, or co-overexpressed either with NtPLC3-RFP or with NtPLC3-RFP H124A in tobacco pollen tubes. Data are from three independent experiments, and $>50$ cells were analyzed for each transformation. Asterisks indicate a significant difference to the AtPIP5K2-EYFP control according to a Student's t-test $\left({ }^{*}, p \leq 0.05 ;{ }^{* * *}, p \leq 0.01\right)$. (B) Fluorescence intensities recorded during the co-overexpression of PIP5K2-EYFP with NtPLC3-RFP or with NtPLC3-RFP H124A. Scale bars $=10 \mu \mathrm{m}$. 


\subsection{NtPLC3-EYFP Displays Discontinuous Plasma Membrane Localization Omitting Circular Nanodomains.}

Our results suggested that NtPLC 3 contributes to the modulation of the PtdIns $(4,5) \mathrm{P}_{2}$ domain in the subapical plasma membrane of pollen tubes. This interpretation is consistent with the initial observation that NtPLC3 decorates an expanded subapical plasma membrane domain compared to PIP5K2 or PIP5K11 (Figure 1). Therefore, the distribution of NtPLC3-EYFP in the subapical plasma membrane of pollen tubes was analyzed in more detail by SD microscopy. When observed by SD microscopy, the NtPLC3-EYFP marker displayed uniform localization throughout the plasma membrane, with the noted exception that fluorescence spared out certain dot-like membrane areas (Figure 5A, B). Intensity profiles such as that recorded along the dashed line in Figure 5B indicate that the plasma membrane fluorescence of NtPLC3-EYFP dropped sharply in the dark dots from gray values of $\sim 200$ units to values below $\sim 75$ units (Figure $5 B$ ). The dark dots with reduced NtPLC3-EYFP fluorescence had diameters of $\sim 0.5 \mu \mathrm{m}$ (Figure 5C) and appeared at a density of $\sim 0.5 \pm 0.2 \mu \mathrm{m}^{-2}$ (Figure 5D). The diameter of the dark dots and their area density were analyzed with increasing distance to the pollen tube tip over the region spanned by NtPLC3. While dot diameters did not change (Figure 5C), there was a minor but significant reduction in dot density with increasing distance from the tip (Figure 5D). A control experiment was performed by analyzing the plasma membrane distribution of PIP5K5-EYFP (Figure 5E, F). PIP5K5-EYFP also localized to the subapical plasma membrane of pollen tubes, but when analyzed by SD microscopy, displayed a uniform distribution in the plane of the plasma membrane (Figure 5F). Overall, the data suggest that NtPLC3 might influence PtdIns $(4,5) \mathrm{P}_{2}$ at the nanodomain scale.

A
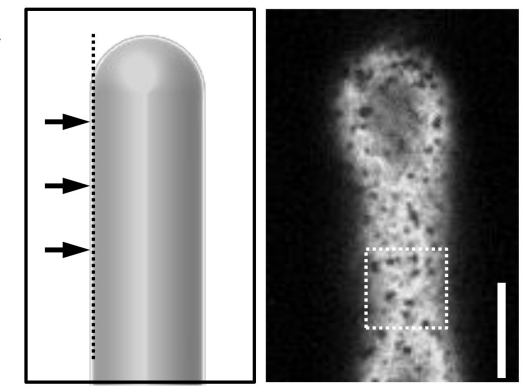

EYFP-NtPLC3
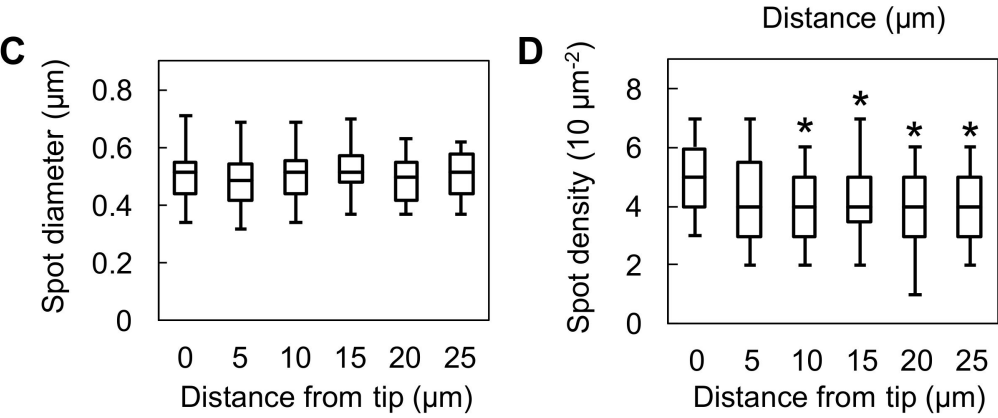

Figure 5. Cont. 


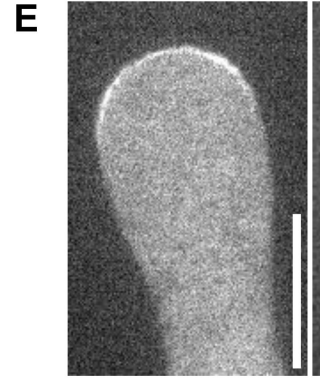

PIP5K5-EYFP

medial

confocal plane

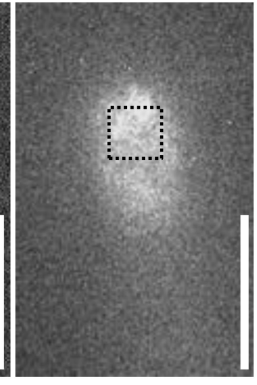

PIP5K5-EYFP

plasma membrane surface

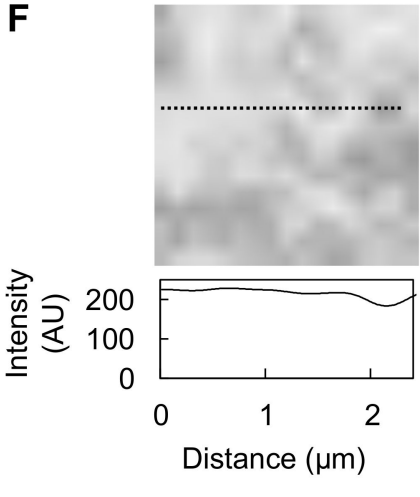

Figure 5. NtPLC3-EYFP localizes in a discontinuous pattern, omitting circular plasma membrane nanodomains. The fluorescence distribution of overexpressed NtPLC3-EYFP in the plasma membrane was assessed by SD microscopy. Only cells displaying low fluorescence intensities were imaged; they exhibited no morphological changes. (A) Association of NtPLC3-EYFP with the pollen tube plasma membrane in a discontinuous pattern omitting circular nanodomains, determined by SD microscopy. The pattern is representative for data from three independent experiments, and 15 cells were analyzed. Scale bar $=5 \mu \mathrm{m}$. (B) Upper panel: Enlargement of the area indicated by the dashed box in (A). Dashed line, trace recorded for intensity analysis. Lower panel: Intensity profile for NtPLC3-EYFP fluorescence along the line. (C) Diameter of NtPLC3-EYFP-excluded domains, analyzed at different distances from the pollen tube tip, as indicated. Data are from three independent experiments, and 25 nanodomains were analyzed per distance; (D) Area density of NtPLC3-EYFP- excluded domains, analyzed at different distances from the pollen tube tip, as indicated. Data are from three independent experiments and 12 areas were analyzed per distance. (E) Association of PIP5K5-EYFP with the pollen tube plasma membrane in a continuous pattern of the plasma membrane, determined by SD microscopy as a control. Left panel, medial confocal section; right panel, peripheral section at the plasma membrane surface. The pattern is representative for data from three independent experiments, and nine cells were analyzed. Scale bar $=5 \mu \mathrm{m}$. (F) Upper panel: Enlargement of the area indicated by the dashed box in (A). Dashed line, trace recorded for intensity analysis. Lower panel: Intensity profile for PIP5K5-EYFP fluorescence along the line. Asterisks indicate a significant difference from the values at distance zero according to a Student's t-test $\left.{ }^{*}, p \leq 0.05\right)$.

\section{Discussion}

The dynamic control of PtdIns $(4,5) \mathrm{P}_{2}$ production in the subapical plasma membrane of pollen tubes is critical for polar cell expansion. In this study, we addressed how pollen tube growth is influenced by the contrapuntal activities of PI4P 5-kinase and PLC, which act in biosynthesis and breakdown of PtdIns $(4,5) \mathrm{P}_{2}$, respectively. Our data indicate that NtPLC3 can functionally antagonize the effects of PtdIns $(4,5) \mathrm{P}_{2}$ formed by PI4P 5-kinases in vivo. This notion is supported by the observations that i) NtPLC3 localizes in a plasma membrane region encompassing that which is occupied by PIP5K2 (Figure 1); ii) the expression of the dominant negative NtPLC3 H124A resulted in similar tip swelling of pollen tubes as the expression of the PI4P 5-kinases PIP5K2 or PIP5K11 (Figure 3); and iii) the expression of NtPLC3, but not of its inactive variant NtPLC3 H124A, abated the effects of the co-overexpression of PIP5K2 on pollen tube morphology (Figure 4). Our SD-based analysis furthermore shows an unexpected fluorescence distribution of NtPLC3 in the subapical plasma membrane of pollen tubes, where the enzyme occupies the majority of the membrane plane but is excluded from small punctate patches resembling plasma membrane "nanodomains" (Figure 5). Together, these findings suggest that NtPLC3 functionally antagonizes PI4P 5-kinases in vivo to modulate PtdIns $(4,5) \mathrm{P}_{2}$ with a key role in the control of pollen tube growth.

It should be noted that the fluorescence patterns or physiological effects observed upon overexpression of PI4P 5-kinases or other enzymes in pollen tubes under the control of strong pollen-specific promoters, such as Lat52 [32], may not reflect the situation in unperturbed cells. 
However, transient expression by particle bombardment can be used to obtain a range of expression intensities, which, for PI4P 5-kinases, were previously be found to correlate with the degree of ensuing morphological alterations of the pollen tube cells. In morphologically normal cells, subcellular distribution patterns obtained for different fluorescence-tagged PI4P 5-kinases by transient Lat52-driven expression have previously been shown to faithfully reflect the patterns found for these fusion proteins in Arabidopsis pollen tubes when expressed from their respective intrinsic promoters in the corresponding Arabidopsis mutant backgrounds [12,13].

Based on the fluorescence distribution of NtPLC3 observed by SD (Figure 5), it is tempting to speculate whether the punctate plasma membrane areas not occupied by NtPLC3 might be sites of enhanced PtdIns $(4,5) \mathrm{P}_{2}$ formation and/or residence of PI4P 5-kinases. In support of this notion, the presence of PtdIns $(4,5) \mathrm{P}_{2}$ nanodomains has previously been proposed, based on the analysis of plant plasma membrane preparations by transmission electron microscopy and immuno gold labeling [33]. Furthermore, small GTPases of the ROP family, which are important for pollen tube growth [10] and are functionally linked to PtdIns $(4,5) \mathrm{P}_{2}[13,17]$, have been observed to reside in membrane nanodomains [34]. PtdIns(4,5) $\mathrm{P}_{2}$ may be restricted to nanodomains by the action of degrading enzymes, such as PLC, as previously proposed [7]. Our model (Figure 6) illustrates a possible role for NtPLC3 in restricting PtdIns $(4,5) \mathrm{P}_{2}$ formation in some areas while enabling PtdIns $(4,5) \mathrm{P}_{2}$ formation in others.

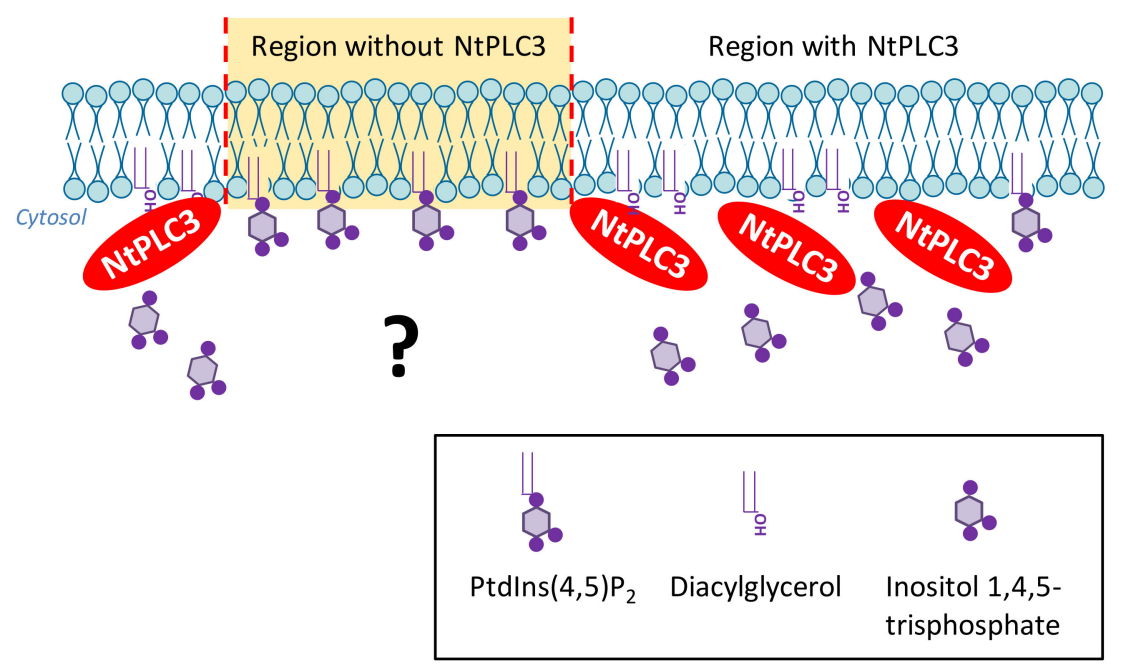

Figure 6. A simplified model of NtPLC3 antagonizing PtdIns(4,5) $\mathbf{P}_{2}$-formation. A possible role for NtPLC3 is in restricting PtdIns $(4,5) \mathrm{P}_{2}$ production in some areas of the plasma membrane while enabling it in other areas. The punctate plasma membrane areas not occupied by NtPLC3-EYFP fluorescence might be sites of enhanced PtdIns $(4,5) \mathrm{P}_{2}$ formation and/or residence of PI4P 5-kinases. PtdIns $(4,5) \mathrm{P}_{2}$ abundance might thus be controlled and restricted to nanodomains by the action of degrading enzymes, such as PLC. Other explanations are possible.

While this model attempts to reflect the data without much speculation, the molecular basis for the interesting localization pattern of NtPLC3 remains currently unclear. It is possible that local asymmetries in the distribution of certain membrane lipids, such as sphingolipids or sterols, and the ensuing differences in the biophysical properties of certain membrane areas, may be important aspects regarding the correct recruitment of NtPLC3 to regions where its activity is required [35-38]. Unfortunately, the analysis of such interrelations by fluorescence microscopy is impeded by the limitations of the biosensors and fluorescent markers that are currently available, which exert a substantial influence on the function of the intrinsic phosphoinositide system in vivo [8,39,40]. With respect to the actual study, the expression of NtPLC3, an active enzyme degrading PtdIns $(4,5) \mathrm{P}_{2}$, will influence the distribution of PtdIns $(4,5) \mathrm{P}_{2}$ biosensors. Reciprocally, the binding of a PtdIns $(4,5) \mathrm{P}_{2}$ 
biosensor to its lipid target might influence the accessibility of the lipid for degradation by PLC [41]. Possibly, for these or related reasons, so far we have not been able to observe meaningful localization patterns of NtPLC3-RFP vs. a coexpressed biosensor for $\operatorname{PtdIns}(4,5) \mathrm{P}_{2}$. It is possible that future advances in biosensor development or in imaging technology may help us to circumvent some of these experimental caveats.

At a larger scale, our study demonstrates antagonistic effects of PI4P 5-kinases and PLC on a relevant physiological process in vivo, and the proposed functional interplay can easily be rationalized from the known in vitro activities of these enzymes. In addition, the data hold a number of unexpected findings, which will require further study in the future to complete the picture. For instance, it remains unclear why the expression of the dominant negative NtPLC3 H124A resulted in tip swelling, but not in any detectable pollen tube tip branching. Both pollen tube tip swelling [13,19] and tip branching $[12,14,19]$ have previously been shown to result from the overproduction of PtdIns $(4,5) \mathrm{P}_{2}$ through different isoforms of PI4P 5-kinases. Our data on the effects of expressed NtPLC3 H124A are consistent with the corresponding results on petunia PLC1, where also only pollen tube tip swelling was observed [21].

Overall, it appears possible that NtPLC3 or petunia PLC1 can antagonize some but not other regulatory pathways mediated by $\operatorname{PtdIns}(4,5) \mathrm{P}_{2}$, possibly as a consequence of a difference in the accessibility or subcompartmentalization of $\operatorname{PtdIns}(4,5) \mathrm{P}_{2}$ formed by different PI4P 5-kinase isoforms in the pollen tube plasma membrane. This notion is consistent with previous results on the functionality of hypervariable linker domains of plant PI4P 5-kinases in mediating membrane recruitment into different regulatory contexts [19]. The effect of $\operatorname{Ptd} \operatorname{Ins}(4,5) \mathrm{P}_{2}$ on pollen tube tip swelling has previously been rationalized through a stabilizing effect of PtdIns $(4,5) \mathrm{P}_{2}$ on the actin cytoskeleton [13]. It remains to be seen whether NtPLC3 might indirectly also influence the dynamics of the actin cytoskeleton in pollen tubes through its effects on $\operatorname{Ptd} \operatorname{Ins}(4,5) \mathrm{P}_{2}$. Future experiments will elucidate whether the antagonistic effects of PLC with PI4P 5-kinases may be relevant for pollen tube functionality, genetic transmission or seed set.

\section{Materials and Methods}

\section{1. cDNA Constructs}

The coding sequence for NtPLC3 was amplified from tobacco cDNA using the following primer combination 5'-GATCCCATGGCATCGAGACAGACGTACAGAGTCT-3'/5'-GATCCCATGGA CATATTCGAAACGCATAAGAAGC-3', and moved as an NcoI/NcoI fragment into the expression plasmid pET M-41, encoding the NtPLC3 protein with an N-terminal maltose binding protein (MBP) tag. Site-directed mutagenesis was performed on the NtPLC3 coding sequence using the primer combination 5'-TCTCATTACTTCATATACACAGGAGCTAATTCCTATCTAACTGGGAATCAA-3'/ 5'-TTGATTCCCAGTTAGATAGGAATTAGCTCCTGTGTATATGAAGTAATGAGA-3', yielding NtPLC3 H124A. For expression as fluorescent fusions, the coding sequences for NtPLC3 or NtPLC3 H124A were subcloned into pEntryD and moved by Gateway technology into the pLat52-GW vector [12] in frame with the coding sequence for RFP. Constructs for the expression of fluorescent fusions of Arabidopsis PIP5K2 [19] or PIP5K11 [13] were used as previously reported.

\subsection{Recombinant Protein Expression}

Protein extracts containing recombinant NtPLC3 and NtPLC3 H124A protein were generated by the expression in E. coli Rosetta 2 cells. Cells were grown in liquid LB media, selecting for kanamycin and chloramphenicol resistance. Expression cultures of $100 \mathrm{ml}$ were incubated at $37^{\circ} \mathrm{C}$ with shaking at $200 \mathrm{rpm}$. Expression was induced at an optical density of 0.8 by adding $1 \mathrm{mM}$ isopropyl- $\beta$-D-thiogalactosylpyranoside (IPTG). Cultures were incubated overnight at $25^{\circ} \mathrm{C}$ with shaking at $200 \mathrm{rpm}$ and harvested by centrifugation. The cell sediment was resuspended on ice in lysis buffer containing $50 \mathrm{mM}$ Tris- $\mathrm{HCl}, \mathrm{pH} 8,300 \mathrm{mM} \mathrm{NaCl}, 1 \mathrm{mM}$ EDTA, $10 \%$ (w/v) glycerol and $50 \mathrm{U}$ of 
lysozyme. Cells were ruptured by ultrasound on ice, using five $60 \mathrm{~s}$ bursts in a Sonifier Cell Disruptor B15 (Branson Dietzenbach, Germany) at 50\% power and 50\% impulse settings. The protein extracts were cleared by centrifugation and stored at $-20^{\circ} \mathrm{C}$. Proteins were analyzed by immunodetection as previously described [42,43].

\subsection{Assay for PLC Activity}

PLC activity was assayed in vitro according to the release of radiolabel from the $2\left[{ }^{3} \mathrm{H}\right] \mathrm{Ptd} \operatorname{Ins}(4,5) \mathrm{P}_{2}$ substrate from an organic to an aqueous solvent phase, as previously described [29]. In brief, $2 \mu \mathrm{Ci}$ of $2\left[{ }^{3} \mathrm{H}\right] \mathrm{PtdIns}(4,5) \mathrm{P}_{2}$ substrate (DuPont/New England Nuclear, Wellesley, MA, USA) was mixed in chloroform with $8 \mu \mathrm{g}$ of unlabeled PtdIns $(4,5) \mathrm{P}_{2}$ (Avanti, Alabaster, AL, USA) at a ratio of 2/8 $(v / v)$ and dried in a glass reaction vial. The lipid coat was redissolved in $5 \mu \mathrm{L}$ of $1 \%(w / v)$ Triton X-100 and sonified on ice in a waterbath sonicator. Recombinant protein extract was added at 5-10 $\mu \mathrm{g}$ in a volume of $30 \mu \mathrm{L}$. Then, $20 \mu \mathrm{L}$ of reaction buffer containing $50 \mathrm{mM}$ Tris- $\mathrm{HCl}, \mathrm{pH} 6.4$, and $20 \mu \mathrm{M}$ $\mathrm{CaCl}_{2}$ were added and the samples incubated at $25^{\circ} \mathrm{C}$ for $10 \mathrm{~min}$. Reactions were stopped by adding $500 \mu \mathrm{L}$ of chloroform:methanol (2:1) $(v / v)$ and $500 \mu \mathrm{L} 1 \mathrm{M} \mathrm{HCl}$. Phases were allowed to separate and the aqueous phase was collected. The radiolabel in the aqueous phase was analyzed with a liquid scintillation counter (LSA Tricarb 1900 TR, Canberra Packard, Dreieich, Germany).

\subsection{Transient Expression of Constructs in Tobacco Pollen Tubes}

Mature pollen grains were collected from four to five tobacco flowers of approximately eightto nine-week-old plants. The pollens were resuspended in liquid pollen tube growth media [44], followed by filtering of the pollen grains onto a cellulose acetate filter, before being transferred onto Whatmann paper which was moistened with pollen tube growth media. They were immediately transformed by bombardment with plasmid-coated 1- $\mu \mathrm{m}$ gold particles with a helium-driven particle accelerator (PDS-1000/He; Bio-Rad) using 1350 psi rupture disks and a vacuum of 28 inches of mercury, as previously described [45]. Prior to the bombardment, gold particles were coated with $4-5 \mu \mathrm{g}$ of the desired plasmid of interest. After bombardment, the pollen grains were transferred into $300 \mu \mathrm{L}$ of pollen tube growth media which was then equally divided onto three microscopic glass slides and viewed under the microscope $4-6 \mathrm{~h}$ after the bombardment.

\subsection{Live Cell Microscopy and Image Processing}

Images were acquired with confocal Zeiss LSM510 meta or Zeiss LSM880 Airyscan systems, or with a Zeiss Cell observer SD with a Yokogawa CSU-X1 SD unit. SD microscopy allowed us to capture confocal imaging information with short dwell-times by using a rotating disc to generate multiple pin-holes in parallel. Short dwell times are additionally enabled by using a camera, which is more light-sensitive than a confocal point scanner, and can capture fluorescence signals of low intensity. For imaging with the LSM880, a 20x or a $63 x$ oil immersion objective was used and images were captured with the ZEN Black image analysis software. During acquisition with the LSM880, EYFP was excited at $514 \mathrm{~nm}$ laser line and imaged with a HFT $514 \mathrm{~nm}$ major beam splitter (MBS) while mCherry or RFP was excited with a 561nm laser line and imaged with a HFT 561nm MBS. Image acquisition with the Zeiss Cell observer SD was performed with a $63 x$ oil immersion objective and captured with a Photometrics Evolve 512 Delta EM-CCD camera. YFP was excited at $491 \mathrm{~nm}$ and mCherry at $561 \mathrm{~nm}$ using a multichannel dichroic and an ET525/50M or an ET595/50M band pass emission filter (Chroma Technology) for GFP and mCherry respectively. The Zen Blue image analysis software was used for image processing. FM 4-64 was added to pollen tubes at a final concentration of $10 \mu \mathrm{M}$, as previously described [46], and visualized after 5-15 min of incubation. Kymographs were obtained using Fiji image analysis software [47] by plotting fluorescence intensities in the region of interest over time. Since pollen tubes exhibit a fast growth rate and their growth is not linear, it was difficult to track the fluorescence intensities values of PLC and PIP5K11 at the cell membrane without substantial manual adjustment. To eliminate possible bias and provide an independent analysis of 
the relative distribution patterns of PLC3 and PIP5K11, the machine learning program Ilastik was used [27] to analyze the intensity and distribution of the PIP5K11 and PLC signals. By using the pixel classification and object classification tool, one time frame was used to train the program. Afterwards, the batch processing function analyzed all the remaining frames with no further manual processing.

\subsection{Statistical Evaluation}

All quantitative data were tested for statistical significance using two-tailed Student's t tests. Confidence intervals are given in the figure legends for each data set.

\subsection{Accession Numbers}

Sequence data from this article can be found in the GenBank/EMBL data libraries under the following accession numbers: AtPIP5K2, At1g77740, AtPIP5K5, At2g41210, AtPIP5K11, At1g01460, NtPLC3 and EF043044

Author Contributions: Conceptualization, I.S., T.I., P.K., M.F., I.H.; investigation, I.S., T.I., L.H.V.-B., M.R., P.K., M.F., I.H.; supervision, I.H.; funding acquisition, I.H.; writing, I.H. All authors have read and agreed to the published version of the manuscript.

Funding: This research was funded by the German Research Foundation (DFG, grants He3424/1-1, He3424/6-1, CRC648 TP B10 and INST271/371-1 FUGG to I.H.).

Acknowledgments: We thank Kirsten Bacia (Martin-Luther-University Halle-Wittenberg, Halle, Germany) for access to the spinning disc microscope. The cDNA clone for PIP5K2-mCherry was kindly provided by Mareike Heilmann (Martin-Luther-University Halle-Wittenberg).

Conflicts of Interest: The authors declare no conflict of interest. The funders had no role in the design of the study; in the collection, analyses, or interpretation of data; in the writing of the manuscript, or in the decision to publish the results.

\section{References}

1. Dresselhaus, T.; Sprunck, S.; Wessel, G.M. Fertilization mechanisms in flowering plants. Curr. Biol. 2016, 26, R125-R139. [CrossRef]

2. Cole, R.A.; Fowler, J.E. Polarized growth: Maintaining focus on the tip. Curr. Opin. Plant Biol. 2006, 9, 579-588. [CrossRef] [PubMed]

3. Heilmann, I.; Ischebeck, T. Male functions and malfunctions: The impact of phosphoinositides on pollen development and pollen tube growth. Plant Reprod. 2016, 29, 3-20. [CrossRef] [PubMed]

4. Hepler, P.K.; Winship, L.J. The pollen tube clear zone: Clues to the mechanism of polarized growth. J. Integr. Plant Biol. 2015, 57, 79-92. [CrossRef] [PubMed]

5. Higashiyama, T.; Takeuchi, H. The mechanism and key molecules involved in pollen tube guidance. Annu. Rev. Plant Biol. 2015, 66, 393-413. [CrossRef]

6. Ischebeck, T.; Seiler, S.; Heilmann, I. At the poles across kingdoms: Phosphoinositides and polar tip growth. Protoplasma 2010, 240, 13-31. [CrossRef]

7. Gerth, K.; Lin, F.; Menzel, W.; Krishnamoorthy, P.; Stenzel, I.; Heilmann, M.; Heilmann, I. Guilt by association: A phenotype-based view of the plant phosphoinositide network. Annu. Rev. Plant Biol. 2017, 68, 349-374. [CrossRef]

8. Heilmann, I. Phosphoinositide signaling in plant development. Development 2016, 143, 2044-2055. [CrossRef]

9. Van Leeuwen, W.; Okresz, L.; Bogre, L.; Munnik, T. Learning the lipid language of plant signalling. Trends Plant Sci. 2004, 9, 378-384. [CrossRef]

10. Kost, B. Spatial control of rho (rac-rop) signaling in tip-growing plant cells. Trends Cell Biol. 2008, 18, 119-127. [CrossRef]

11. Heilmann, M.; Heilmann, I. Plant phosphoinositides-complex networks controlling growth and adaptation. Biochim. Biophys. Acta 2015, 1851, 759-769. [CrossRef] [PubMed]

12. Ischebeck, T.; Stenzel, I.; Heilmann, I. Type b phosphatidylinositol-4-phosphate 5-kinases mediate pollen tube growth in nicotiana tabacum and arabidopsis by regulating apical pectin secretion. Plant Cell 2008, 20, 3312-3330. [CrossRef] [PubMed] 
13. Ischebeck, T.; Stenzel, I.; Hempel, F.; Jin, X.; Mosblech, A.; Heilmann, I. Phosphatidylinositol-4,5-bisphosphate influences nt-rac5-mediated cell expansion in pollen tubes of nicotiana tabacum. Plant J. 2011, 65, 453-468. [CrossRef] [PubMed]

14. Sousa, E.; Kost, B.; Malho, R. Arabidopsis phosphatidylinositol-4-monophosphate 5-kinase 4 regulates pollen tube growth and polarity by modulating membrane recycling. Plant Cell 2008, 20, 3050-3064. [CrossRef]

15. Zhao, Y.; Yan, A.; Feijo, J.A.; Furutani, M.; Takenawa, T.; Hwang, I.; Fu, Y.; Yang, Z. Phosphoinositides regulate clathrin-dependent endocytosis at the tip of pollen tubes in arabidopsis and tobacco. Plant Cell 2010, 22, 4031-4044. [CrossRef]

16. Ischebeck, T.; Vu, L.H.; Jin, X.; Stenzel, I.; Löfke, C.; Heilmann, I. Functional cooperativity of enzymes of phosphoinositide conversion according to synergistic effects on pectin secretion in tobacco pollen tubes. Mol. Plant 2010, 3, 870-881. [CrossRef]

17. Kost, B.; Lemichez, E.; Spielhofer, P.; Hong, Y.; Tolias, K.; Carpenter, C.; Chua, N.H. Rac homologues and compartmentalized phosphatidylinositol 4,5-bisphosphate act in a common pathway to regulate polar pollen tube growth. J. Cell Biol. 1999, 145, 317-330. [CrossRef]

18. Heilmann, I. Plant phosphoinositide signaling—dynamics on demand. Biochim. Biophys. Acta 2016, 1861, 1345-1351. [CrossRef]

19. Stenzel, I.; Ischebeck, T.; Quint, M.; Heilmann, I. Variable regions of pi4p 5-kinases direct ptdins $(4,5) \mathrm{p} 2$ toward alternative regulatory functions in tobacco pollen tubes. Front. Plant Sci. 2012, 2, 1-14. [CrossRef]

20. Helling, D.; Possart, A.; Cottier, S.; Klahre, U.; Kost, B. Pollen tube tip growth depends on plasma membrane polarization mediated by tobacco plc3 activity and endocytic membrane recycling. Plant Cell 2006, 18, 3519-3534. [CrossRef]

21. Dowd, P.E.; Coursol, S.; Skirpan, A.L.; Kao, T.H.; Gilroy, S. Petunia phospholipase c1 is involved in pollen tube growth. Plant Cell 2006, 18, 1438-1453. [CrossRef] [PubMed]

22. Pokotylo, I.; Kolesnikov, Y.; Kravets, V.; Zachowski, A.; Ruelland, E. Plant phosphoinositide-dependent phospholipases c: Variations around a canonical theme. Biochimie 2014, 96, 144-157. [CrossRef] [PubMed]

23. Mähs, A.; Ischebeck, T.; Heilig, Y.; Stenzel, I.; Hempel, F.; Seiler, S.; Heilmann, I. The essential phosphoinositide kinase mss-4 is required for polar hyphal morphogenesis, localizing to sites of growth and cell fusion in neurospora crassa. PLoS ONE 2012, 7, e51454. [CrossRef] [PubMed]

24. Ischebeck, T.; Werner, S.; Krishnamoorthy, P.; Lerche, J.; Meijon, M.; Stenzel, I.; Löfke, C.; Wiessner, T.; Im, Y.J.; Perera, I.Y.; et al. Phosphatidylinositol 4,5-bisphosphate influences pin polarization by controlling clathrin-mediated membrane trafficking in arabidopsis. Plant Cell 2013, 25, 4894-4911. [CrossRef]

25. Mei, Y.; Jia, W.J.; Chu, Y.J.; Xue, H.W. Arabidopsis phosphatidylinositol monophosphate 5-kinase 2 is involved in root gravitropism through regulation of polar auxin transport by affecting the cycling of pin proteins. Cell Res. 2012, 22, 581-597. [CrossRef]

26. Tejos, R.; Sauer, M.; Vanneste, S.; Palacios-Gomez, M.; Li, H.; Heilmann, M.; van Wijk, R.; Vermeer, J.E.; Heilmann, I.; Munnik, T.; et al. Bipolar plasma membrane distribution of phosphoinositides and their requirement for auxin-mediated cell polarity and patterning in arabidopsis. Plant Cell 2014, 26, 2114-2128. [CrossRef]

27. Berg, S.; Kutra, D.; Kroeger, T.; Straehle, C.N.; Kausler, B.X.; Haubold, C.; Schiegg, M.; Ales, J.; Beier, T.; Rudy, M.; et al. Ilastik: Interactive machine learning for (bio)image analysis. Nat. Methods 2019, 16, 1226-1232. [CrossRef]

28. Heilmann, M.; Heilmann, I. Arranged marriage in lipid signalling? The limited choices of ptdins $(4,5) \mathrm{p}$ in finding the right partner. Plant Biol. 2013, 15, 789-797. [CrossRef]

29. Hirayama, T.; Ohto, C.; Mizoguchi, T.; Shinozaki, K. A gene encoding a phosphatidylinositol-specific phospholipase $\mathrm{c}$ is induced by dehydration and salt stress in arabidopsis thaliana. Proc. Natl. Acad. Sci. USA 1995, 92, 3903-3907. [CrossRef]

30. Hernandez-Sotomayor, S.M.; Munoz-Sanchez, J.A. Determination of phospholipase c activity in vitro. Methods Mol. Biol. 2013, 1009, 187-192.

31. Ischebeck, T. Distinct roles of pi4p 5-kinases in polar growth of pollen tubes. Ph.D. Thesis, Georg-August-University Göttingen, Göttingen, Germany, 2008.

32. Twell, D.; Yamaguchi, J.; McCormick, S. Pollen-specific gene expression in transgenic plants: Coordinate regulation of two different tomato gene promoters during microsporogenesis. Development 1990, 109, 705-713. [PubMed] 
33. Furt, F.; Konig, S.; Bessoule, J.J.; Sargueil, F.; Zallot, R.; Stanislas, T.; Noirot, E.; Lherminier, J.; Simon-Plas, F.; Heilmann, I.; et al. Polyphosphoinositides are enriched in plant membrane rafts and form microdomains in the plasma membrane. Plant Physiol. 2010, 152, 2173-2187. [CrossRef] [PubMed]

34. Platre, M.P.; Bayle, V.; Armengot, L.; Bareille, J.; Marques-Bueno, M.D.M.; Creff, A.; Maneta-Peyret, L.; Fiche, J.B.; Nollmann, M.; Miege, C.; et al. Developmental control of plant rho gtpase nano-organization by the lipid phosphatidylserine. Science 2019, 364, 57-62. [CrossRef] [PubMed]

35. Markham, J.E.; Molino, D.; Gissot, L.; Bellec, Y.; Hematy, K.; Marion, J.; Belcram, K.; Palauqui, J.C.; Satiat-Jeunemaitre, B.; Faure, J.D. Sphingolipids containing very-long-chain fatty acids define a secretory pathway for specific polar plasma membrane protein targeting in arabidopsis. Plant Cell 2011, 23, 2362-2378. [CrossRef] [PubMed]

36. Men, S.; Boutte, Y.; Ikeda, Y.; Li, X.; Palme, K.; Stierhof, Y.D.; Hartmann, M.A.; Moritz, T.; Grebe, M. Sterol-dependent endocytosis mediates post-cytokinetic acquisition of pin2 auxin efflux carrier polarity. Nat. Cell Biol. 2008, 10, 237-244. [CrossRef]

37. Willemsen, V.; Friml, J.; Grebe, M.; van den Toorn, A.; Palme, K.; Scheres, B. Cell polarity and pin protein positioning in arabidopsis require sterol methyltransferase1 function. Plant Cell 2003, 15, 612-625. [CrossRef]

38. Skirpan, A.L.; Dowd, P.E.; Sijacic, P.; Jaworski, C.J.; Gilroy, S.; Kao, T.H. Identification and characterization of piorp1, a petunia oxysterol-binding-protein related protein involved in receptor-kinase mediated signaling in pollen, and analysis of the orp gene family in arabidopsis. Plant Mol. Biol. 2006, 61, 553-565. [CrossRef]

39. Balla, T.; Bondeva, T.; Varnai, P. How accurately can we image inositol lipids in living cells? Trends Pharmacol. Sci. 2000, 21, 238-241. [CrossRef]

40. Balla, T.; Varnai, P. Visualizing cellular phosphoinositide pools with gfp-fused protein-modules. Sci. STKE 2002, 2002, pl3. [CrossRef]

41. Drobak, B.K.; Watkins, P.A.C.; Valenta, R.; Dove, S.K.; Lloyd, C.W.; Staiger, C.J. Inhibition of plant plasma membrane phosphoinositide phospholipase $\mathrm{c}$ by the actin-binding protein, profilin. Plant J. 1994, 6, 389-400. [CrossRef]

42. Hempel, F.; Stenzel, I.; Heilmann, M.; Krishnamoorthy, P.; Menzel, W.; Golbik, R.; Helm, S.; Dobritzsch, D.; Baginsky, S.; Lee, J.; et al. Mapks influence pollen tube growth by controlling the formation of phosphatidylinositol 4,5-bisphosphate in an apical plasma membrane domain. Plant Cell 2017, 29, 3030-3050. [CrossRef] [PubMed]

43. Menzel, W.; Stenzel, I.; Helbig, L.M.; Krishnamoorthy, P.; Neumann, S.; Eschen-Lippold, L.; Heilmann, M.; Lee, J.; Heilmann, I. A pamp-triggered mapk cascade inhibits phosphatidylinositol 4,5-bisphosphate production by pip5k6 in arabidopsis thaliana. N. Phytol. 2019, 224, 833-847. [CrossRef] [PubMed]

44. Read, S.M.; Clarke, A.E.; Bacic, A. Stimulation of growth of cultured nicotiana tabacum w 38 pollen tubes by poly(ethylene glycol) and cu(ii) salts. Protoplasma 1993, 177, 1-14. [CrossRef]

45. Muller, A.O.; Blersch, K.F.; Gippert, A.L.; Ischebeck, T. Tobacco pollen tubes-a fast and easy tool for studying lipid droplet association of plant proteins. Plant J. 2017, 89, 1055-1064. [CrossRef] [PubMed]

46. Parton, R.M.; Fischer-Parton, S.; Watahiki, M.K.; Trewavas, A.J. Dynamics of the apical vesicle accumulation and the rate of growth are related in individual pollen tubes. J. Cell Sci. 2001, 114, 2685-2695.

47. Schindelin, J.; Arganda-Carreras, I.; Frise, E.; Kaynig, V.; Longair, M.; Pietzsch, T.; Preibisch, S.; Rueden, C.; Saalfeld, S.; Schmid, B.; et al. Fiji: An open-source platform for biological-image analysis. Nat. Methods 2012, 9, 676-682. [CrossRef]

(C) 2020 by the authors. Licensee MDPI, Basel, Switzerland. This article is an open access article distributed under the terms and conditions of the Creative Commons Attribution (CC BY) license (http://creativecommons.org/licenses/by/4.0/). 